Hogg: Does the star suspected of being in the stage of contraction exhibit emission lines in its spectrum?

Thackeray: None have been detected.

Oort: In answer to the question by Dr. Thackeray, one could say that the disruption of a large cloud complex by $\mathrm{O}$-type stars could easily take longer than a million years. With expansion velocities of $10 \mathrm{~km} / \mathrm{sec}$ the gas would move a distance of the order of $10 \mathrm{pc}$ in a million years. But if the mass of gas in the association is sufficiently large the expansional velocities might become very small before the density boundary is reached, so that a spread of star formation over a period of, say, $10^{7} \mathrm{yr}$ appears easily possible.

\title{
5. A DISCUSSION OF NGC 4755 AND SOME OTHER YOUNG CLUSTERS IN THE GALAXY AND THE MAGELLANIC CLOUDS
}

\author{
M. W. Feast \\ Radcliffe Observatory
}

A study of the cluster NGC 4755 ( $\kappa$ Crucis) was begun as a result of the several interesting problems raised by an earlier study of NGC 3293 (Feast 1958). Determinations of radial velocity and spectral type have been made in NGC 4755 from 102 spectra of 35 stars. Full details are published elsewhere (Feast 1963). These results were combined with the three-colour photometry of Arp and van Sant (1958). The cluster has a nearly-vertical evolved main sequence at spectral type Bl. The spectral types and $B, V$ measures for giants and dwarfs yield a mean reddening of $+0 \mathrm{~m} 48$ and a distance of $2 \cdot 36 \mathrm{kpc}$. These values are considerably greater than those deduced by Arp and van Sant but they have been confirmed from an analysis of the threecolour measurements alone.

NGC 4755 contains an M-type supergiant, four B-type supergiants, and possibly also an A-type supergiant. This is a valuable addition to the number of clusters known to contain such stars and thus to be of known absolute magnitude and intrinsic colour. The available data for B-type supergiants in clusters have been examined. In the mean the absolute magnitudes agree well with Blaauw's (1963) calibration, but the intrinsic colours are about 0m04 bluer than those given by Johnson (1958). Two clusters in Cygnus give anomalous results, probably due to an abnormal reddening law in this direction.

The HR diagrams of NGC 4755, NGC 3293, and $\mathrm{h}$ and $\chi$ Per are very similar and these three clusters must be of nearly the same age. A composite HR diagram for the three clusters together contains a sufficient density of points for certain of the finer details to be seen. There is a conspicuous gap between the top of the evolved main sequence and the supergiant branch. A gap in an analogous position was first discovered in the rich somewhat-older clusters NGC 458 and NGC 330 in the SMC by Arp (1959). At the time, this gap seemed to constitute an important difference between clusters in the Galaxy and those in the SMC. However, the present results make it quite clear, as had been suspected from the results on $h$ and $\chi$ Per alone (Feast 1960), that the gap is also a feature of clusters in the Galaxy. Indeed, a recent theoretical investigation (Hayashi and Cameron 1962) predicts that such a gap should exist.

NGC 4755, NGC 3293, and $\mathrm{h}$ and $\chi$ Per contain M-type supergiants but no supergiants of intermediate type. On the other hand, the evidence from other clusters 
and also the region near the Sun is that for somewhat fainter supergiants there is a much higher frequency of intermediate types. That the type-frequency plot (Hess diagram) for supergiants may be rather sensitive to absolute magnitude is important, amongst other things, because some comparisons between clusters in the Galaxy and in the Magellanic Clouds have been essentially comparisons of Hess diagrams for supergiants. Thus Hodge (1961) contrasted the Hess diagram of NGC 1844 in the LMC with that of NGC 4755. However, the greater distance for NGC 4755 adopted in the present work removes the similarity in the absolute magnitudes of supergiants in the two clusters.

The previous work on NGC 3293 (Feast 1958) had indicated that the measured radial velocities of the giants were more negative than those of the supergiants or the dwarfs. The present work on NGC 4755 and the published data on $h$ and $\chi$ Per yield similar results. The three clusters together give a mean difference in radial velocity between 13 B-type supergiants and 13 B-type giants of $+7 \cdot 3 \pm 1 \cdot 6$ (s.e.) $\mathrm{km} / \mathrm{sec}$. The difference between the supergiants and the dwarfs is negligible $(+1 \cdot 8$ $\pm 2 \cdot 2$ (s.e.) $\mathrm{km} / \mathrm{sec}$ ). The difference between the giants and supergiants may be related to the Trumpler effect and is perhaps attributable to the effects of convective turbulence in the stellar atmospheres. The phenomenon may affect discussions of the galactic motions of B-type stars and further work on it is planned.

\section{References}

ARP, H. C. (1959).-A.J. 64: 175-82 and 254-8.

ARP, H. C., and vaN SANT, C. T. (1958).-A.J. 63 : 341-6.

BlaAUW, A. (1963).--In "Stars and Stellar Systems." (General Ed. G. P. Kuiper.) Vol. III. (Univ. Chicago Press: in press.)

Feast, M. W. (1958).-M.N. 118: 618-30.

Feast, M. W. (1960).—Observatory 80: 104-6.

Feast, M. W. (1963).-M.N. 126: 11-28.

HAYASH, C., and CAMERON, R. C. (1962).—Ap.J. $136: 166-92$.

HODGe, P. W. (1961).-Ap. J. 134: 226-31.

JoHNson, H. L. (1958).-Lowell Obs. Bulletin 4: 37-46.

\section{Discussion}

Araham : I have some fragmentary observations for the $\kappa$ Crucis cluster in the form of $\mathrm{H} \beta$ indices for five of the bright stars which support the distance derived by Feast. On adopting Feast's value of the reddening for the cluster these indicate a distance of $2100 \mathrm{pc} \pm$ about $400 \mathrm{pc}$.

Hogg: Preliminary results of $U B V$ photoelectric photometry in NGC 4755 have given a reddening of 0.41 and a distance of $2 \mathrm{kpc}$, in fair agreement with the results stated by $\mathrm{Dr}$. Feast.

Walraven: The gap between the top of the main sequence and the supergiant branch observed by Dr. Feast is also present in the colour-magnitude diagrams of both the Small and Large Magellanic Clouds.

Westerlund: I derived the distance modulus $m-M=11 \cdot 8$ for the $\kappa$ Crucis cluster some years ago using my own $U B V$ observations of the blue supergiants and Bidelman's spectral classification. Later on I combined i.r. photometry with Arp's and van Sant's BV photometry. Some of the fainter stars may possibly be contracting stars. I also identified two Be stars in the cluster. Have they been studied spectroscopically and where do they fall in the new diagram?

Feast: One cannot be certain that all stars not on the main sequence are indeed foreground stars. Two Be stars were included, but they were not noticably displaced in the HR diagram. 